\title{
Integrated PVM Framework Supports Heterogeneous Network Computing
}

Jack J. Dongarra

Oak Ridge National Laboratory and University of Tennessee

\author{
G. A. Geist \\ Oak Ridge National Laboratory \\ Robert Manchek \\ University of Tennessee \\ V. S. Sunderam \\ Emory University
}

January 3, 1993

\begin{abstract}
The Parallel Virtual Machine (PVM), an integrated framework for heterogeneous network computing, lets scientists exploit collections of networked machines when carrying out complex scientific computations. Under PVM, a user-defined grouping of serial, parallel, and vector computers appears as one large distributed-memory machine. Configuring a personal parallel virtual computer involves simply listing the names of the machines in a file that is read when PVM is started. Applications can be written in Fortran 77 or $\mathrm{C}$ and parallelized by use of message-passing constructs common to most distributed-memory computers. With the use of messages sent over the network, multiple tasks of an application can cooperate to solve a problem in parallel.

This article discusses components of PVM, including the programs and library of interface routines. It summarizes the characteristics of appropriate applications and discusses the current status and availability of PVM. In addition, the article introduces a recent extension to PVM known as the Heterogeneous Network Computing Environment (HeNCE).
\end{abstract}




\section{Introduction}

Two developments promise to revolutionize scientific problem solving. The first is the development of massively parallel computers. Massively parallel systems offer the enormous computational power needed for solving Grand Challenge problems. Unfortunately, software development has not kept pace with hardware advances. In order to fully exploit the power of these massively parallel machines, new programming paradigms, languages, scheduling and partitioning techniques, and algorithms are needed.

The second major development affecting scientific problem solving is distributed computing. Many scientists are discovering that their computational requirements are best served not by a single, monolithic machine but by a variety of distributed computing resources, linked by high-speed networks.

Heterogeneous network computing offers several advantages: By using existing hardware the cost of this computing can be very low. Performance can be optimized by assigning each individual task to the most appropriate architecture. Network computing also offers the potential for partitioning a computing task along lines of service functions. Typically, networked computing environments possess a variety of capabilities; the ability to execute subtasks of a computation on the processor most suited to a particular function both enhances performance and improves utilization. Another advantage in network-based concurrent computing is the ready availability of development and debugging tools, and the potential fault tolerance of the network(s) and the processing elements. Typically, systems that operate on loosely coupled networks permit the direct use of editors, compilers, and debuggers that are available on individual machines. These individual machines are quite stable, and substantial expertise in their use is readily available. These factors translate into reduced development and debugging time and effort for the user, and reduced contention for resources and possibly more effective implementations of the application. Yet another attractive feature of loosely coupled computing environments is the potential for userlevel or program-level fault tolerance that can be implemented with little effort either in the application or in the underlying operating system. Most multiprocessors do not support such a facility; hardware or software failures in one of the processing elements often lead to a complete crash.

Despite the advantages of heterogeneous network computing, however, many issues remain to be addressed. Of especial importance are issues relating to the user interface, efficiency, compatibility, and administration. In 
some cases, individual researchers have attempted to address these issues by developing ad hoc approaches to the implementation of concurrent applications. Recognizing the growing need for a more systematic approach, several research groups have recently attempted to develop programming paradigms, languages, scheduling and partitioning techniques, and algorithms.

Our approach is more pragmatic. We discuss the development of an integrated framework for heterogeneous network computing, in which a collection of interrelated components provides a coherent high-performance computing environment. In particular, we analyze several of the design features of the PVM (Parallel Virtual Machine) system. Figure 1 gives an overview of the system.

The paper is organized as follows. In Section 2, we give a brief look at the general field of heterogeneous network computing and discuss some of the research issues remaining before network-based heterogeneous computing is truly effective. In Section 3, we focuses on the PVM system, which is designed to help scientists write programs for such heterogeneous systems. In Section 4, we discuss a recent extension of PVM that further aids in the implementation of concurrent applications.

\section{Connecting Heterogeneous Computers}

In the past, researchers have conducted experiments linking workstations that provide on the order of 1 to 10 MIPS. Such experiments have included remote execution, computer farms, and migration of computations.

More recently, experiments have focused on linking higher-performance workstations (those providing on the order of 10 to 100 MFLOPS) together with multiprocessors and conventional supercomputers.

To fully exploit these multiple computer configurations, researchers have developed various software packages that enable scientists to write truly heterogeneous programs. Examples of such software packages include Express, P4, Linda, and PVM. Each package is layered over the native operating systems, exploits distributed concurrent processing, and is flexible and generalpurpose; all exhibit comparable performance. Their differences lie in their programming model, their implementation schemes, and their efficiency.

Recent conferences as well as informal discussion media seem to indicate that most attention is focused on the four systems mentioned aboveExpress, P4, Linda, and PVM-in terms of use by the high performance sci- 
entific computing community. In the remainder of this section, we present brief outlines of each of the first three, with a detailed description of PVM following in Section 3. We wish to emphasize however, (1) that these systems are by no means the only software packages in existence; and (2) the descriptions that follow are not detailed and formal critiques, but rather brief synopses abstracted from our understanding of the systems and the developers' own articles or communications.

\subsection{The Linda Model and System}

Linda [9] is a concurrent programming model that has evolved from a Yale University research project. The primary concept in Linda is that of a "tuple-space", an abstraction via which cooperating processes communicate. This central theme of Linda has been proposed as an alternative paradigm to the two traditional methods of parallel processing, viz. that based on shared memory, and on message passing. The tuple-space concept is essentially an abstraction of distributed shared memory, with one important difference (tuple-spaces are associative), and several minor distinctions (destructive and non-destructive reads, and different coherency semantics are possible). Applications use the Linda model by embedding explicitly, within cooperating sequential programs, constructs that manipulate (insert/retrieve tuples) the tuple space.

From the application point of view Linda [4] is a set of programming language extensions for facilitating parallel programming. The Linda model is a scheme built upon an associative memory referred to as tuple-space It provides a shared memory abstraction for process communication without requiring the underlying hardware to physically share memory. The model is illustrated in Figure 2 [9].

Tuples are collections of fields logically "welded" to form persistent storage items. They are the basic tuple-space storage units. Parallel processes exchange data by generating, reading, and consuming them. To update a tuple, the tuple is removed from tuple-space, modified, and returned to tuple-space. Restricting tuple-space modification in this manner creates an implicit locking mechanism ensuring proper synchronization of multiple accesses.

The following are the four basic operations or primitives which are added to a language to produce a Linda dialect. Figure 2 depicts the operational environment when using Linda.

(1) $r d(t)$ performs a non-destructive read from tuple-space. If the desired 
tuple, "t", is not found, the invoking process is suspended until an appropriate tuple is created by another process.

(2) in(t) behaves in a fashion similar to $\mathrm{rd}()$, except the read is destructive and the tuple is consumed.

(3) out (t) writes a tuple "t" to tuple-space.

(4) eval(expression) writes a tuple to tuple-space after arguments in the expression are evaluated by creating new processes which perform their tasks independently.

Tuples are selected by the rd() or in() primitives on the basis of their field values. There are no tuple addresses in an associative memory. Consider the following tuple:

$$
\text { out("a string", 15.01, 17, "another string") }
$$

A variety of access routes to this tuple are possible, e.g., any one of the following operations suffices:

$$
\begin{aligned}
& \text { rd("a string", ?fval, ?ival, ?strval) } \\
& \text { rd(?strval, 15.01, ?ival, "another string") } \\
& \text { rd(?strval-1, ?fval, 17, ?strval-2) }
\end{aligned}
$$

The "?" operator designates a value returned from a matching tuple. Fields marked by the operator do not participate in the (associative memory) matching process. Any of the three example rd() operations results in a nondestructive reading of the original tuple. If the operation were an in(), the tuple would be removed from tuple-space.

To illustrate the eval() primitive, consider the following:

$$
\text { eval("roots",sqrt(4),sqrt(16)) }
$$

Using Linda terminology, this creates a live tuple. The square-root operations are performed independent of the originating process, with the (two) numeric results combined to form a three element tuple saved in tuplespace. The eval() primitive is a mechanism capable of creating fine grain parallelism. 
The "Linda System" usually refers to a specific (sometimes portable) implementation of software that supports the Linda programming model. System software is provided that establishes and maintains tuple spaces, that is used in conjunction with libraries that appropriately interpret and execute Linda primitives. Depending on the environment (shared memory multiprocessors, message passing parallel computers, networks of workstations etc), the tuple space mechanism is implemented using different techniques, and with varying degrees of efficiency. Recently, a new system technique has been proposed, at least nominally related to the Linda project. This scheme, termed "Pirhana" proposes a proactive approach to concurrent computing the idea being that computational resources (viewed as active agents) seize computational tasks from a well known location based on availability and suitability. Again, this scheme may be implemented on multiple platforms, and manifested as a "Pirhana system" or "Linda-Pirhana system".

\section{$2.2 \quad$ P4 and Parmacs}

P4 is a library of macros and subroutines developed at Argonne National Laboratory for programming a variety of parallel machines. The P4 system supports both the shared-memory model (based on monitors) and the distributed-memory model (using message-passing). For the shared-memory model of parallel computation, $\mathrm{P} 4$ provides a set of primitives from which monitors can be constructed, as well as a set of useful monitors. For the distributed-memory model, $\mathrm{P} 4$ provides typed send and receive operations, and creation of processes according to a text file describing group and process structure. P4 is intended to be portable, simple to install and use, and efficient. It can be used to program networks of workstations, advanced parallel supercomputers like the Intel Touchstone Delta and the Alliant Campus HiPPI-based system, and single shared-memory multiprocessors. It has currently been installed on most uniprocessor workstations, shared memory multiprocessors, and several high-performance parallel machines.

Process management in the $\mathrm{P} 4$ system is based on a configuration file that specifies the host pool, the object file to be executed on each machine, the number of processes to be started on each host (intended primarily for multiprocessor systems) and other auxiliary information. An example of a configuration file is

\# start one slave on each of sun2 and sun3

local 0 


\section{sun2 1 /home/mylogin/p4pgms/sr_test}

sun3 1 /home/mylogin/p4pgms/sr_test

Two issues are noteworthy in regard to the process management mechanism in P4. First, there is the notion a "master" process and "slave" processes, and multilevel hierarchies may be formed to implement what is termed a cluster model of computation. Second, the primary mode of process creation is static, via the configuration file; dynamic process creation is possible only by a statically created process that must invoke a special $\mathrm{P} 4$ function that spawns a new process on the local machine. However, despite these restrictions, a variety of application paradigms may be implemented in the $\mathrm{P} 4$ system in a fairly straightforward manner.

Message Passing in the P4 system is achieved through the use of traditional send and recv primitives, parameterized almost exactly as other message passing systems. Several variants are provided for semantics such as heterogeneous exchange, and blocking or nonblocking transfer. A significant proportion of the burden of buffer allocation and management however, is left to the user. Apart from basic message passing, $\mathrm{P} 4$ also offers a variety of global operations, including broadcast, global maxima and minima, and barrier synchronization.

Shared Memory support via monitors is a facility that distinguishes P4 from other systems. However, this feature is not distributed shared memory; but rather, a portable mechanism for shared address space programming in true shared memory multiprocessors. The abstraction provided by $\mathrm{P} 4$ for managing data in shared memory is monitors. The specific approach taken by $\mathrm{P} 4$ is described in [3]. P4 provides several useful monitors (p4_barrier_t, p4_getsub_monitor_t, p4_askfor_monitor_t) as well as a general monitor type to help the user in constructing his own monitors (p4_monitor_t).

P4 also supports a variety of auxiliary and support functions, for timing purposes and for debugging. The latter set of functions are essentially printing facilities that identify the source of a debugging message, and "levels" of debugging are provided so that the user may control the volume of debugging information that is printed. Finally, the $\mathrm{P} 4$ system also contains a package (ALOG) for creating logs of time-stamped events, that is of general utility, outside of $\mathrm{P} 4$. The timestamps are obtained from various microsecond-level resolution timers on various machines. These $\log$ files are primarily intended for use with a separate tool termed Upshot [5] that visually depicts events and their ordering from a $\mathrm{P} 4$ application run. 
Parmacs is a project that is closely related to the P4 effort. Essentially, Parmacs is a set of macro extensions to the P4 system developed at GMD [6]. It originated in an effort to provide Fortran interfaces to the P4 system, but is now a significantly enhanced package that provides a variety of high-level abstractions, mostly dealing with global operations. Parmacs provides macros for logically configuring a set of $\mathrm{P} 4$ processes; for example, the macro torus produces a suitable configuration file for use by $\mathrm{P} 4$ that results in a logical process configuration corresponding to a 3 - $d$ torus. Other logical topologies, including general graphs may also be implemented, and Parmacs provides macros used in conjunction with send and recv to achieve topology-specific communications within executing programs.

\section{$2.3 \quad$ Express}

In contrast to the other parallel processing systems described in this section, Express toolkit is a collection of tools that individually address various aspects of concurrent computation. The toolkit is developed and marketed commercially by ParaSoft Corporation, a company that was started by some members of the Caltech concurrent computation project. A second distinction is support for PC's that Express provides, in addition to the usual high performance computing platforms and workstations.

The philosophy behind computing with Express is based on beginning with a sequential version of an application and following a recommended development life cycle culminating in a parallel version that is tuned for optimality. Typical development cycles begin with the use of VTOOL, a graphical program that allows the progress of sequential algorithms to be displayed in a dynamic manner. Updates and references to individual data structures can be displayed to explicitly demonstrate algorithm structure and provide the detailed knowledge necessary for parallelization. Related to the above is FTOOL, which provides in-depth analysis of a program including variable use analysis, flow structure and feedback regarding potential parallelization. FTOOL operates on both sequential and parallel versions of an application. A third tool called ASPAR is then used; this is an auto-

mated parallelizer that converts sequential $\mathrm{C}$ and FORTRAN programs for parallel or distributed execution using the Express programming models.

The core of the Express system is a set of libraries for communication, IO, and parallel graphics. The communication primitives are akin to those found in other systems, and include a variety of global operations and data distribution primitives. Extended IO routines enable parallel input and 
output, and a similar set of routines are provided for graphical displays from multiple concurrent processes. Express also contains the NDB tool, a parallel debugger that used commands which are based on the popular "dbx" interface. These debugging commands can be issued to single processors or groups of nodes simultaneously.

Express finally contains a set of "back-end" tools intended to assist performance monitoring and tuning. CTOOL analyzes high level overhead issues such as the relative amount of time spent computing, performing IO and in interprocessor communication. ETOOL shows the relationships between various computing elements and may be used to understand overheads, and cause and effect relationships between actions in different processors. XTOOL profiles CPU usage on a per-processor basis, and may be used at different levels of granularity.

\subsection{Ongoing Trends}

In the next section of this paper, we focus on the basic features of PVM and discuss our experiences with that system. PVM as well as the systems described above have evolved over the past several years, but none of them can be considered fully mature. The field of network based concurrent computing is relatively young, and research on various aspects is ongoing. Although basic infrastructures have been developed, many of the refinements that are necessary are still evolving. Some of the ongoing research projects related to heterogeneous network-based computing are briefly outlined here.

Standalone systems delivering several tens of millions of operations per second are commonplace, and continuing increases in power are predicted. For network computing systems, this presents many challenges. One aspect concerns scaling to hundreds and perhaps thousands of independent ma-

chines; it is conjectured that functionality and performance equivalent to massively parallel machines can be supported on cluster environments. A project at Fermilab has demonstrated the feasibility of scaling to hundreds of processors for some classes of problems. Research in protocols to support scaling and other system issues are currently under investigation. Further, under the right circumstances, the network based approach can be effective in coupling several similar multiprocessors, resulting in a configuration that might be economically and technically difficult to achieve with hardware.

Applications with large execution times will benefit greatly from mechanisms that make them resilient to failures. Currently few platforms (especially among multiprocessors) support application level fault tolerance. In a 
network based computing environment application resilience to failures can be supported without specialized enhancements to hardware or operating systems. Research is in progress to investigate and develop strategies for enabling applications to run to completion, in the presence of hardware, system software, or network faults. Approaches based on checkpointing, shadow execution, and process migration are being investigated.

The performance and effectiveness of network based concurrent computing environments depends to a large extent on the efficiency of the support software, and on minimization of overheads. Experiences with the PVM system have identified several key factors in the system that are being further analyzed and improved to increase overall efficiency. Efficient protocols to support high level concurrency primitives is a subgoal of work in this area. Particular attention is being given to exploiting the full potential of imminent fiber optic connections, using an experimental fiber network that is available. In preliminary experiments with a fiber optic network, several important issues have been identified. For example, the operating system interfaces to fiber networks, its reliability characteristics, and factors such as maximum packet size are significantly different from those for Ethernet. When the concurrent computing environment is executed on a combination of both types of networks, the system algorithms have to be modified to cater to these differences, in an optimal manner and with minimized overheads.

Another issue to be addressed concerns data conversions that are necessary in networked heterogeneous systems. Heuristics to perform conversions only when necessary and minimizing overheads have been developed and their effectiveness is being evaluated. Recent experiences with a Cray-2 have also identified the need to handle differences in wordsize and precision, when operating in a heterogeneous environment; general mechanisms to deal with arbitrary precision arithmetic (when desired by applications) are also being developed. A third aspect concerns the efficient implementation of inherently expensive parallel computing operations such as barrier synchronization. Particularly in an irregular environment (where interconnections within hardware multiprocessors are much faster than network channels), such operations can cause bottlenecks and severe load imbalances. Other distributed primitives for which algorithm development and implementation strategies are being investigated include polling, distributed fetch-and-add, global operations, automatic data decomposition and distribution, and mutual exclusion. 


\section{PVM}

PVM [2] was produced by the Heterogeneous Network Project-a collaborative effort by researchers at Oak Ridge National Laboratory, the University of Tennessee, and Emory University specifically to facilitate heterogeneous parallel computing. PVM was one of the first software systems to enable machines with widely different architectures and floating-point representations to work together on a single computational task. It can be used on its own or as a foundation upon which other heterogeneous network software can be built.

The PVM package is small (about than 1 Mbytes of $\mathrm{C}$ source code) and easy to install. It needs to be installed only once on each machine to be accessible to all users. Moreover, the installation does not require special privileges on any of the machines and thus can be done by any user.

The PVM user-interface requires that all message data be explicitly typed. PVM performs machine-indepen dent data conversions when required, thus allowing machines with different integer and floating-point representations to pass data.

\subsection{Various Levels of Heterogeneity}

PVM supports heterogeneity at the application, machine, and network level. At the application level, subtasks can exploit the architecture best suited to the their solution. At the machine level, computers with different data formats are supported as well as different serial, vector, and parallel architectures. At the network level, different network types can make up a Parallel Virtual Machine, for example, Ethernet, FDDI, token ring, etc. Under PVM, a user-defined collection of serial, parallel, and vector computers appears as one large distributed-memory computer; we use the term virtual machine to designate this logical distributed-memory computer. The hardware that composes the user's personal PVM may be any Unix-based machine on which the user has a valid login and that is accessible over some network.

Using PVM, users can also configure their own parallel virtual machine, which can overlap with other users' virtual machines. Configuring a personal parallel virtual machine involves simply listing the names of the machines in a file that is read when PVM is started. Applications, which can be written in Fortran 77 or C, can be parallelized by using message-passing constructs common to most distributed-memory computers. By sending and receiving 
messages, multiple tasks of an application can cooperate to solve a problem in parallel.

PVM supplies the functions to automatically start up tasks on the virtual machine and allows the tasks to communicate and synchronize with each other. In particular, PVM handles all message conversion that may be required if two computers use different data representations. PVM also includes many control and debugging features in its user-friendly interface. For instance, PVM ensures that error messages generated on some remote computer get displayed on the user's local screen.

\subsection{Components of PVM}

The PVM system is composed of two parts. The first part is a daemon, called pvmds, that resides on all the computers making up the virtual computer. (An example of a daemon program is sendmail, which handles all the incoming and outgoing electronic mail on a Unix system.) pvmds is designed so that any user with a valid login can install this daemon on a machine. When a user wishes to run a PVM application, he executes pvmds on one of the computers which in turn starts up pvmd 3 on each of the computers making up the user-defined virtual machine. A PVM application can then be started from a Unix prompt on any of these computers.

The second part of the system is a library of PVM interface routines. This library contains user-callable routines for passing messages, spawning processes, coordinating tasks, and modifying the virtual machine. Application programs must be linked with this library to use PVM.

\subsection{Applications}

Application programs that use PVM are composed of subtasks at a moderately high level of granularity. The subtasks can be generic serial codes, or they can be specific to a particular machine. In PVM, resources may be accessed at three different levels: the transparent mode in which subtasks are automatically located at the most appropriate sites, the architecturedependent mode in which the user may indicate specific architectures on which particular subtasks are to execute, and the machine-specific mode in which a particular machine may be specified. Such flexibility allows different subtasks of a heterogeneous application to exploit particular strengths of individual machines on the network.

Applications access PVM resources via a library of standard interface 
routines. These routines allow the initiation and termination of processes across the network, as well as communication and synchronization between processes. Communication constructs include those for the exchange of data structures as well as high-level primitives such as broadcast, barrier synchronization, and event synchronization.

Application programs under PVM may possess arbitrary control and dependency structures. In other words, at any point in the execution of a concurrent application, the processes in existence may have arbitrary relationships between each other; furthermore, any process may communicate and/or synchronize with any other.

\title{
3.4 Grand Challenge Application Experiences with PVM
}

Over the past few years a number of applications have been developed using PVM. The table below list some of the applications.

\author{
Materials Science \\ Global Climate Modeling \\ Atmospheric, oceanic, and space studies \\ Meterorological forcasting \\ 3 -D groundwater modeling \\ Weather modeling \\ Superconductivity, molecular dynamics \\ Monte Carlo CFD application \\ 2-D and $3-\mathrm{D}$ seismic imaging \\ 3-D underground flow fields \\ Particle simulation \\ Distributed AVS flow visualization
}

These implementations have been done on various platforms.

During the last few years, ORNL material scientists and their collaborators at the University of Cincinnati, SERC at Daresbury, and the University of Bristol have been developing an algorithm for studying the physical properties of complex substitutionally disordered materials. A few important examples of physical systems and situations in which substitutional disorder plays a critical role in determining material properties include: high-strength alloys, high-temperature superconductors, magnetic phase transitions, and metal/insulator transitions. The algorithm being developed is an implementation of the Korringa, Kohn and Rostoker coherent potential approximation (KKR-CPA) method for calculating the electronic properties, energetics and 
other ground state properties of substitutionally disordered alloys [10]. The KKR-CPA method extends the usual implementation of density functional theory (LDA-DFT) [11] to substitutionally disordered materials [7]. In this sense it is a completely first principles theory of the properties of substitutionally disordered materials requiring as input only the atomic numbers of the species making up the solid.

The KKR-CPA algorithm contains several locations where parallelism can be exploited. These locations correspond to integrations in the KKRCPA algorithm. Evaluating integrals typically involves the independent evaluation of a function at different locations and the merging of these data into a final value. The integration over energy was parallelized. The parallel implementation is based on a master/slave paradigm to reduce memory requirements and synchronization overhead. In the implementation one processor is responsible for reading the main input file, which contains the number of nodes to be used on each multiprocessor as well as the number and type of workstations to include, the problem description, and the location of relevant data files. This master processor also manages dynamic load balancing of the tasks through a simple pool-of-tasks scheme.

Using PVM the KKRCPA code is able to achieve over 200 Mflops utilizing a network of ten IBM RS/6000 workstations. Given this capability, the KKRCPA code is being used as a research code to solve important materials science problems. Since its development the KKRCPA code has been used to compare the electronic structure of two high temperature superconductors, $\mathrm{Ba}\left(\mathrm{Bi}_{.3} \mathrm{~Pb}_{.7}\right) \mathrm{O}_{3}$ and $\left(\mathrm{Ba}_{.6} \mathrm{~K}_{.4}\right) \mathrm{BiO}_{3}$, to explain anomalous experimental results from a high strength alloy, $\mathrm{NiAl}$, and to study the effect of magnetic multilayers in $\mathrm{CrV}$ and CrMo alloys for their possible use in magnetic storage devices.

The goal of the groundwater modeling group is to develop state of the art parallel models for today's high performance parallel computers, which will enable researchers to model flow with higher resolution and greater accuracy than ever before. As a first step researchers at ORNL have developed a parallel 3-D finite element code called PFEM that models water flow through saturated-unsaturated media. PFEM solves the system of equations

$$
F \frac{\partial h}{\partial t}=\nabla \cdot\left[K_{s} K_{r}(\nabla h+\nabla z)\right]+q,
$$

where $h$ is the pressure head, $t$ is time, $K_{s}$ is the saturated hydraulic conductivity tensor, $K_{r}$ is the relative hydraulic conductivity or relative permeability, $z$ is the potential head, $q$ is the source/sink and $F$ is the water 
capacity ( $F=d \theta / d h$, with $\theta$ the moisture content) after neglecting the compressibility of the water and of the media.

Parallelization was accomplished by partitioning the physical domain and statically assigning subdomains to tasks. The present version uses only static load-balancing and relies on the user to define the partitioning. In each step of the solution the boundary region of each subdomain is exchanged with its neighboring regions.

Originally developed on an Intel iPSC/860 multiprocessor, a PVM version of PFEM was straightforward to create requiring an undergraduate student less than 3 weeks to complete. Presently, the PVM version of PFEM has been delivered to several members of the groundwater modeling group for validation testing using networks of workstations while they await the availability of parallel supercomputers.

\section{Current Status and Availability}

PVM was publicly released in March 1991 and has gone through a number of updates. The present version of the software, Version 3.0, has been tested with various combinations of the following machines: Sun 3, SPARCstation, Microvax, DECstation, IBM RS/6000, HP-9000, Silicon Graphics IRIS, NeXT, Sequent Symmetry, Alliant FX, IBM 3090, Intel iPSC/860, Thinking Machines CM-2, KSR-1, Convex, and CRAY Y-MP. Figure 3 gives a complete list of machines PVM has been ported to.

Version 3.0 has a number of improvements over the previous version (2.4). A list of new features are itemize below.

- Runs on Multiprocessors - Paragon, CM-5, etc. using efficient vendor specific calls underneath

- Dynamic Process Groups - user defined grouping

- Dynamic Configuration - able to add and delete hosts

- Multiple Message Buffers - for interface and library

- Improved Routines - receive by source or type automatic multiple spawns with debug and trace options pack and unpack messages using a stride

- Signal handling - PVM processes can pass and catch 
- New naming convention for routines, (backwards compatability with PVM2.4 is supplied).

PVM is available through netlib. To obtain a description of PVM's features, such as a copy of the PVM User's Guide or source code, one simply sends e-mail to netlib@ornl.gov with the message send index from pvm.

\section{$5 \quad$ Future Directions}

The Heterogeneous Network Project is currently building a second package, called HeNCE (for Heterogeneous Network Computing Environment) [1], on top of PVM.

HeNCE simplifies the task of writing, compiling, running, debugging, and analyzing programs on a heterogeneous network. The goal is (1) to make network computing accessible to scientists and engineers without the need for extensive training in parallel computing and (2) to enable them to use resources best suited for a particular phase of the computation.

In $\mathrm{HeNCE}$, the programmer is responsible for explicitly specifying parallelism by drawing graphs which express the dependencies and control flow of a program. Figure 4 provides an example. HeNCE provides a class of graphs as a usable yet flexible way for the programmer to specify parallelism. The user directly inputs the graph using a graph editor which is part of the HeNCE environment. Each node in a HeNCE graph represents a subroutine written in either Fortran or C. Arcs in the HeNCE graph represent dependencies and control flow. An arc from one node to another represents the fact that the tail node of the arc must run before the node at the head of the arc. During the execution of a HeNCE graph, procedures are automatically executed when their predecessors, as defined by dependency arcs, have completed. Functions are mapped to machines based on a user defined cost matrix.

The focus of this work is to provide a paradigm and graphical support tool for programming a heterogeneous network of computers as a single resource. HeNCE is the graphical based parallel programming paradigm. In HeNCE the programmer explicitly specifies parallelism of a computation by drawing graphs. The nodes in a graph represent user defined subroutines and the edges indicate parallelism and control flow. The HeNCE programming environment consists of a set of graphical modes which aid in the creation, compilation, execution, and analysis of HeNCE programs. The main components consist of a graph editor for writing HeNCE programs, 
a build tool for creating executables, a configure tool for specifying which machines to use, an executioner for invoking executables, and a trace tool for analyzing and debugging a program run. These steps are integrated into a window based programming environment as shown in Figure 5.

An initial version of $\mathrm{HeNCE}$ has recently been made available through netlib. To obtain a description of its features, one should send e-mail to netlib@ornl.gov with the message send index from hence.

Both PVM and HeNCE offer researchers a powerful means for attacking scientific computational problems through heterogeneous network computing. Continued research and development will ensure that this new area meets the needs of scientific computing in the 1990s and beyond.

\section{References}

[1] A. Beguelin, J. Dongarra, G. Geist, R. Manchek, and V. Sunderam, "Solving Computational Grand Challenges Using a Network of Supercomputers." Proceedings of the Fifth SIAM Conference on Parallel Processing, Danny Sorensen, ed., SIAM, Philadelphia, 1991.

[2] A. Beguelin, J. J. Dongarra, G. A. Geist, R. Manchek, and V. S. Sunderam. A Users' Guide to PVM Parallel Virtual Machine. Technical Report ORNL/TM-11826, Oak Ridge National Laboratory, July 1991.

[3] J. Boyle, et. al., Portable Programs for Parallel Processors. Holt, Rinehart, and Winston, 1987.

[4] D. Gelernter, "Domesticating Parallelism", IEEE Computer, 19(8):1216, August 1986.

[5] V. Herrarte and E. Lusk, Studying Parallel Program Behavior with Upshot, Argonne National Laboratory, Technical Report ANL-91/15, 1991.

[6] R. Hempel The ANL/GMD MAcros (Parmacs) in Fortran for Portable Parallel Programming Using Message Passing, GMD Technical Report, November 1991.

[7] D. D. Johnson, D. M. Nicholson, F. J. Pinski, B. L. Györffy, G. M. Stocks, Total energy and pressure calculations for random substitutional alloys, Phys. Rev. B, Vol. 41, 9701 (1990). 
[8] A. Kolawa, "The Express Programming Environment", "The Express Programming Environment", Workshop on Heterogeneous NetworkBased Concurrent Computing, Tallahassee, October 1991.

[9] L. Patterson, et. al., "Construction of a Fault-Tolerant Distributed Tuple-Space", 1993 Symposium on Applied Computing, Indianapolis, February 1993.

[10] G. M. Stocks, W. M. Temmerman, B. L. Györffy Complete solution of the Korringa-Kohn-Rostoker coherent potential approximation: $\mathrm{Cu}-\mathrm{Ni}$ alloys, Phys. Rev. Letter, Vol. 41, 339 (1978).

[11] Ulf von Barth Density Functional Theory for Solids, Electronic structure of complex systems, ed. Phariseau and Temmerman, NATO ASI Series, Plenum Press, (1984). 


\section{SIDEBAR ON Message Passing Interface Forum}

During the past year there has been quite a bit of activity in the community to develop a standard interface for message passing [1]. The main advantages of establishing a message passing standard are portability and ease-of-use. In a distributed memory communication environment in which the higher level routines and/or abstractions are built upon lower level message passing routines the benefits of standardization are particularly apparent. Furthermore, the definition of a message passing standard provides vendors with a clearly defined base set of routines that they can implement efficiently, or in some cases provide hardware support for, thereby enhancing scalability. The standards activity goes by the name Message Passing Interface Forum (MPI Forum) and is composed of the major hardware and software vendors, as well as researchers from universities and laboratories around the world.

The goal of the Message Passing Interface simply stated is to develop a standard for writing message-passing programs. As such the interface should establishing a practical, portable, efficient, and flexible standard for message passing.

A complete list of goals follow.

- Design an application programming interface (not necessarily for compilers or a system implementation library).

- Allow efficient communication: Avoid memory to memory copying and allow overlap of computation and communication and offload to communication coprocessor, where available.

- Allow (but no mandate) extensions for use in heterogeneous environment.

- Allow convenient C, Fortran 77, Fortran 90, and $\mathrm{C}++$ bindings for interface.

- Provide a reliable communication interface: User need not cope with communication failures. Such failures are dealt by the underlying communication subsystem.

- Focus on a proposal that can be agreed upon in 6 months.

- Define an interface that is not too different from current practice, such as PVM, Express, Parmacs, etc. 
- Define an interface that can be quickly implemented on many vendor's platforms, with no significant changes in the underlying communication and system software.

- The interface should not contain more functions than are really necessary.

This standard is intended for use by all those who want to write portable message-passing programs in Fortran 77 and/or C. This includes individual application programmers, developers of software designed to run on parallel machines, and creators of higher-level programming languages, environments, and tools. In order to be attractive to this wide audience, the standard must provide a simple, easy-to-use interface for the basic user while not semantically precluding the high-performance message-passing operations available on advanced machines.

The standard includes (this is temporarily as inclusive as possible):

- Point-to-point communication in a variety of modes, including modes that allow fast communication and heterogeneous communication

- Collective operations

- Process groups

- Communication contexts

- A simple way to create processes for the SPMD model

- Bindings for both Fortran and $\mathrm{C}$

- A model implementation

- A formal specification.

One of the objectives of the activity is to have a definition completed by the Summer 1993. If you are interested in finding out more about the MPI effort contact David Walker (walker@msr.epm.ornl.gov) at Oak Ridge National Laboratory. 


\section{References}

[1] Jack J. Dongarra, Rolf Hempel, Anthony J. G. Hey, and David W. Walker. A Proposal for a User-Level, Message-Passing Interface in a Distributed Memory Environment Technical Report ORNL/TM-??, Oak Ridge National Laboratory, 1992. 
Figure 1: Linda Environment

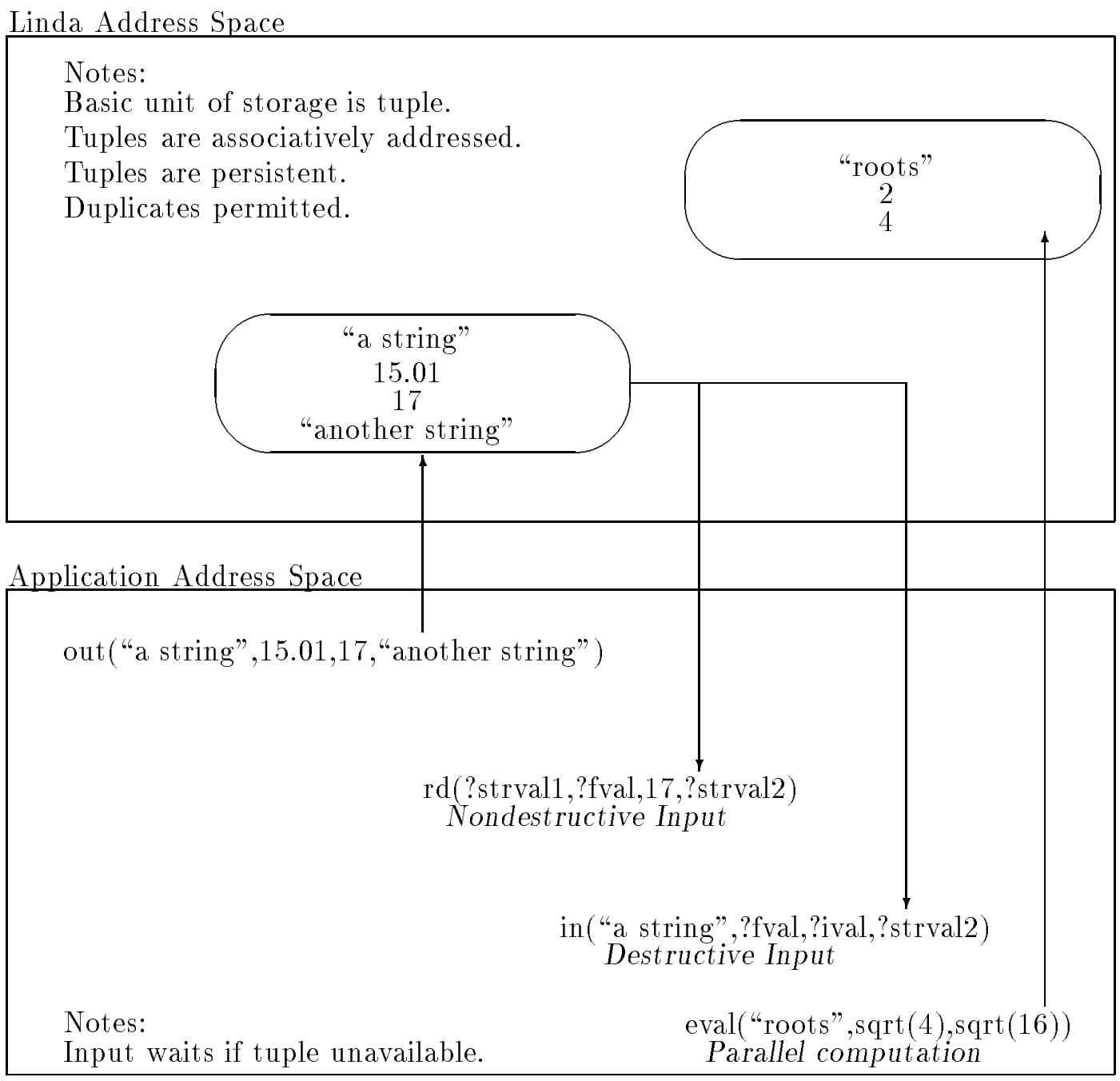

